\title{
An Efficient and Accurate Technique for the Incident-Wave Excitations in the FDTD Method
}

\author{
Uğur Oğuz, Student Member, IEEE, Levent Gürel, Senior Member, IEEE, and Orhan Arıkan, Member, IEEE
}

\begin{abstract}
An efficient technique to improve the accuracy of the finite-difference time-domain (FDTD) solutions employing incident-wave excitations is developed. In the separate-field formulation of the FDTD method, any incident wave may be efficiently introduced to the three-dimensional (3-D) computational domain by interpolating from a one-dimensional (1-D) incident-field array (IFA), which is a 1-D FDTD grid simulating the propagation of the incident wave. By considering the FDTD computational domain as a sampled system and the interpolation operation as a decimation process, signal-processing techniques are used to identify and ameliorate the errors due to aliasing. The reduction in the error is demonstrated for various cases. This technique can be used for the excitation of the FDTD grid by any incident wave. A fast technique is used to extract the amplitude and the phase of a sampled sinusoidal signal.
\end{abstract}

Index Terms-Aliasing, decimation, electromagnetic scattering, FDTD, incident-field array, incident-field excitation, interpolation, sampling.

\section{INTRODUCTION}

$\mathbf{T}$ HE finite-difference time-domain (FDTD) method [1]-[6] is an efficient, flexible, robust, and easy-to-implement technique to solve time-dependent Maxwell's equations. The FDTD method did not receive much interest when it was first suggested three decades ago [1]. With the increase of computing power available to the scientists in recent years, the FDTD method has become one of the most popular solution techniques in the area of computational electromagnetics.

Electromagnetic scattering problems, where the objects are placed in unbounded media and illuminated by incident waves of various types, are among the wide variety of problems solved by using the FDTD method [7]. Therefore, capabilities have been added to the FDTD method to simulate the propagation of the incident waves in unbounded media and their interaction with the scatterers. In this paper, we will investigate the errors introduced to the FDTD solution through incident-wave excitations and present an efficient technique to reduce these errors. Although the usefulness of this technique will be demonstrated using plane-wave excitations with sinusoidal time dependence, the applicability of the technique is not limited by plane waves or waves with sinusoidal time dependence. The technique presented in this

Manuscript received April 28, 1997; revised March 3, 1998.

The authors are with the Department of Electrical and Electronics Engineering, Bilkent University, TR-06533, Bilkent, Ankara, Turkey (e-mail: lgurel@ee.bilkent.edu.tr; oarikan@ee.bilkent.edu.tr).

Publisher Item Identifier S 0018-9480(98)04055-1. paper can be used for the excitation of the FDTD grid by any incident wave.

\section{INCIDENT-WAVE EXCITATION SCHEMES}

In [8] and [19], we discussed in detail various excitation schemes for the FDTD solutions of scattering problems. We addressed the merits of the total-field, scattered-field, and separate-field formulations [9]-[11] in a comparative manner. We also compared the closed-form incident field (CFIF) versus the incident-field array (IFA) excitation schemes. In this paper, we will use the IFA scheme in conjunction with the separatefield formulation.

The IFA is an efficient method of computing incident fields, which was first proposed by Taflove [5], and applied in [8] and [19] with some improvements. The IFA is an FDTDbased look-up table, from which incident-field values are interpolated. The look-up table is a one-dimensional (1-D) grid excited by a hard source, on which the incident wave is propagated by the 1-D FDTD equations. This source grid or IFA is assumed to point in the direction of propagation of the incident wave, as shown in Fig. 1(a). When an incident-field value has to be computed at a particular point in the threedimensional (3-D) computational domain, the relative position of that point is first determined on the source grid. Then, the desired incident-field value is interpolated from the 1-D vector elements. Fig. 1(a) depicts the case of linear interpolation using the closest two points in the source grid, as originally suggested by Taflove [5], although cubic interpolation is used in this paper for improved accuracy [8]. The efficiency of the IFA scheme is due to the fact that both the 1-D FDTD propagation in the IFA and the interpolation operations on the connecting boundary require simple multiplications and additions instead of the evaluation of complicated expressions.

An accurate excitation of the 3-D grid by the 1-D IFA requires the equalization of the numerical phase velocities in the two grids [5], [8]. This is achieved by modifying the material parameters used in the 1-D FDTD equations as

$$
\begin{aligned}
H_{\mathrm{inc}, m+1 / 2}^{n+1 / 2}= & H_{\mathrm{inc}, m+1 / 2}^{n-1 / 2}+\frac{\Delta t}{\Delta \mu_{0}\left[\frac{\tilde{v}_{p}(\theta=0, \phi=0)}{\tilde{v}_{p}(\theta, \phi)}\right]} \\
& \cdot\left(E_{\mathrm{inc}, m}^{n}-E_{\mathrm{inc}, m+1}^{n}\right)
\end{aligned}
$$




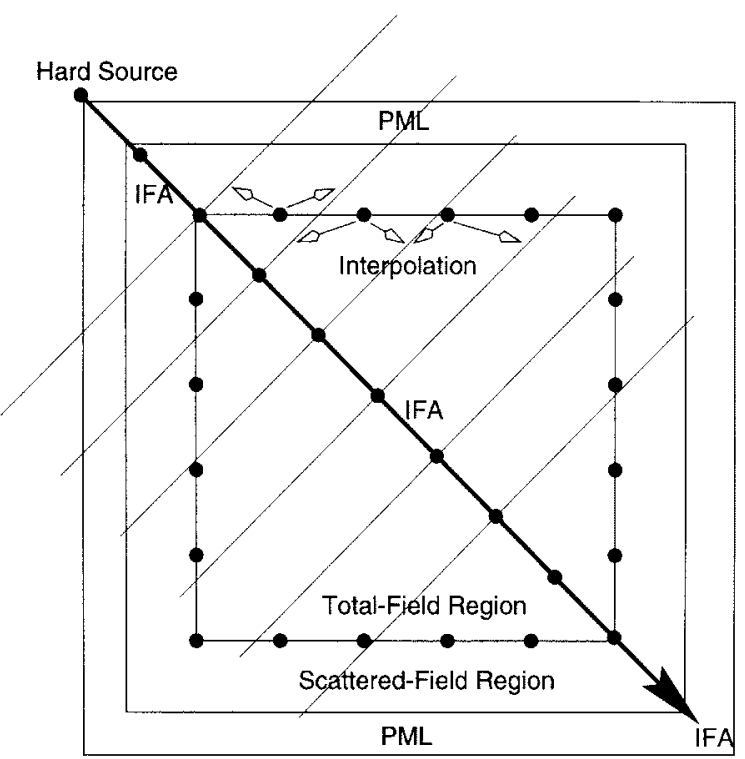

(a)

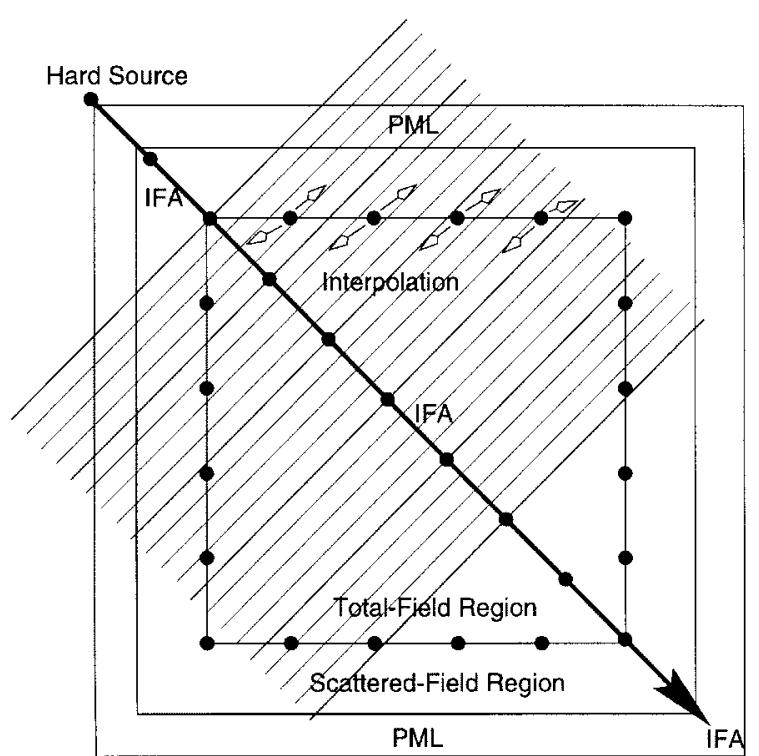

(b)

Fig. 1. (a) The standard IFA excitation scheme in the separate-field formulation. The 1-D source grid (IFA) points in the direction of propagation. The incident-field values in the 3-D computational domain are interpolated from the closest two elements of the 1-D source grid (when linear interpolation is used). (b) Increasing the sampling rate in the source grid. The elements of the 1-D grid are much closer to the point of interest in the 3-D computational domain.

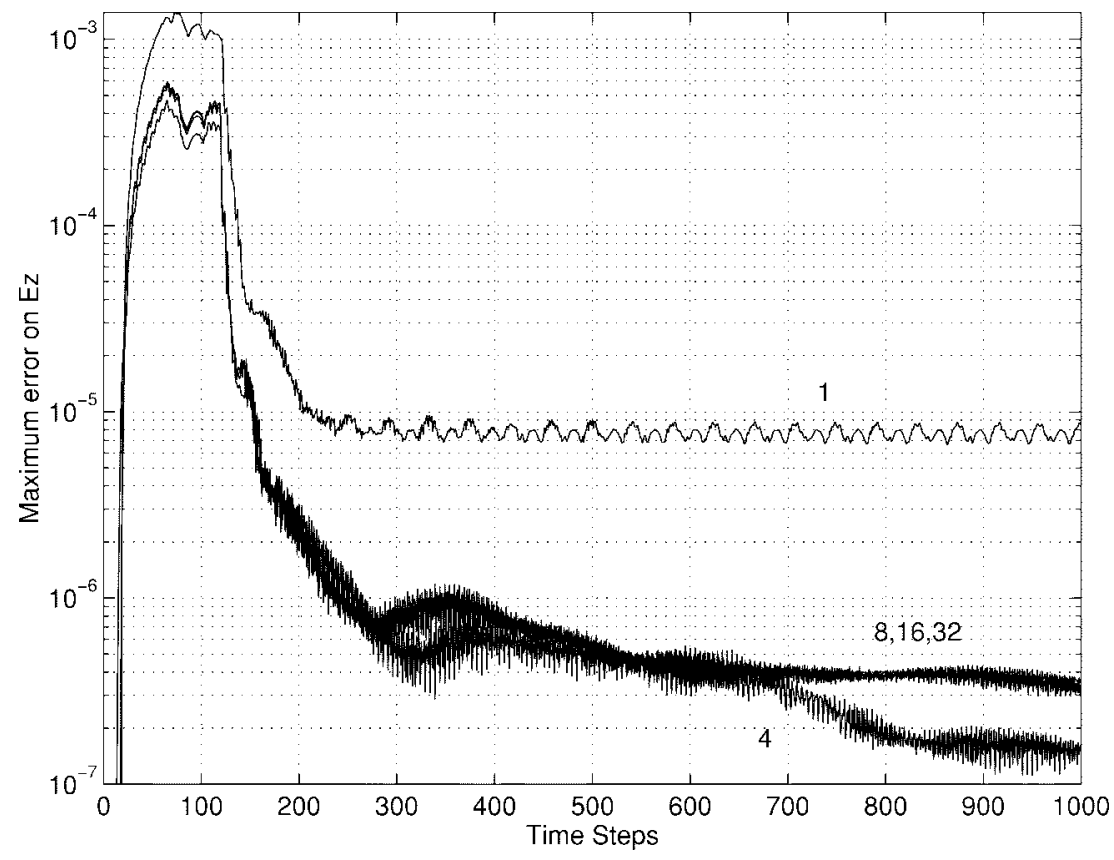

Fig. 2. The maximum errors on $E_{z}$ for 4-, 8-, 16-, and 32-times-better sampled IFA excitations together with the standard IFA excitation. A half-period-long Hanning window is used for smoothing in all five computations.

$$
\begin{aligned}
& E_{\text {inc }, m}^{n+1}= E_{\text {inc }, m}^{n}+\frac{\Delta t}{\Delta \epsilon_{0}\left[\frac{\tilde{v}_{p}(\theta=0, \phi=0)}{\tilde{v}_{p}(\theta, \phi)}\right]} \\
& \cdot\left(H_{\text {inc }, m-1 / 2}^{n+1 / 2}-H_{\text {inc }, m+1 / 2}^{n+1 / 2}\right)
\end{aligned}
$$

where $\tilde{v}_{p}(\theta, \phi)$ is the direction-dependent numerical phase velocity in the $3-\mathrm{D}$ grid, which is related to the numerical wavenumber $\tilde{k}$ through

$$
\tilde{v}_{p}(\theta, \phi)=\omega_{0} / \tilde{k}(\theta, \phi)
$$

The numerical wavenumber $\tilde{k}$ satisfies the discretized dispersion relation

$$
\begin{aligned}
& {\left[\frac{1}{c \Delta t} \sin \left(\frac{\omega \Delta t}{2}\right)\right]^{2}} \\
& \quad=\left[\frac{1}{\Delta x} \sin \left(\frac{\tilde{k}_{x} \Delta x}{2}\right)\right]^{2}+\left[\frac{1}{\Delta y} \sin \left(\frac{\tilde{k}_{y} \Delta y}{2}\right)\right]^{2} \\
& \quad+\left[\frac{1}{\Delta z} \sin \left(\frac{\tilde{k}_{z} \Delta z}{2}\right)\right]^{2}
\end{aligned}
$$



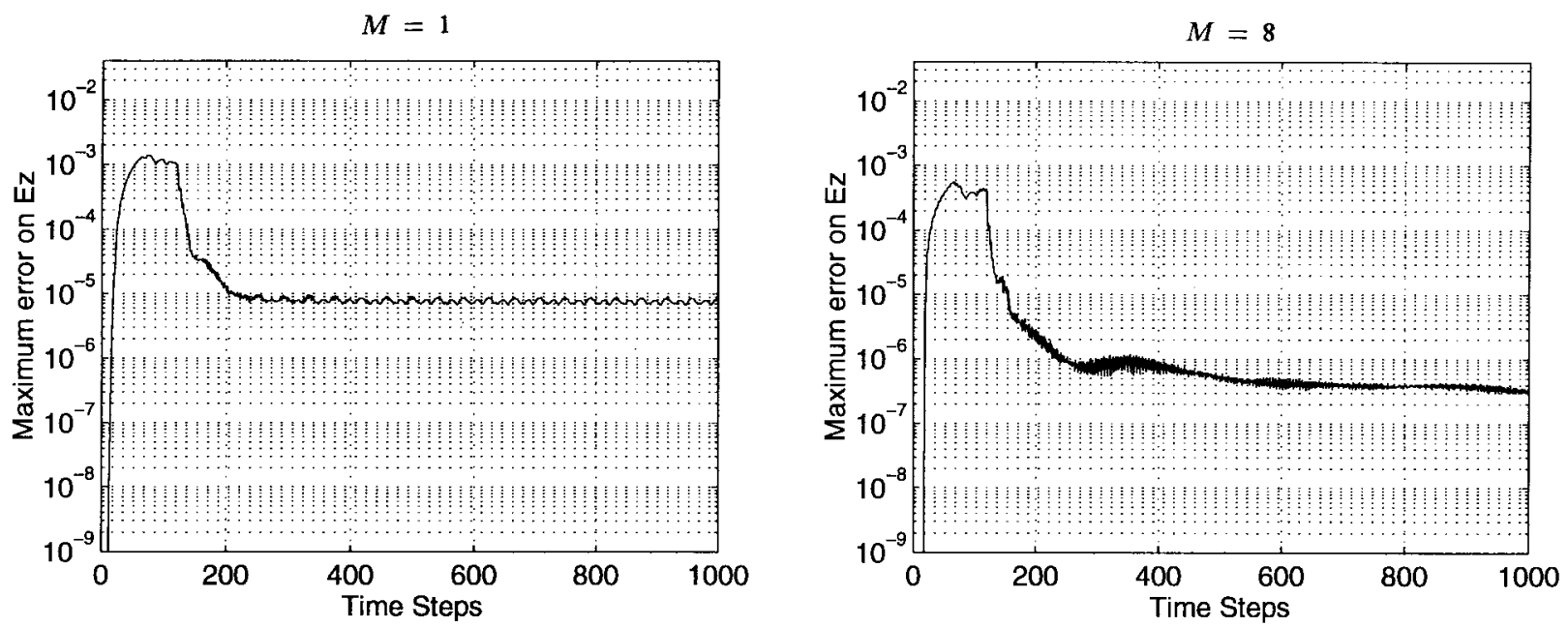

(a)
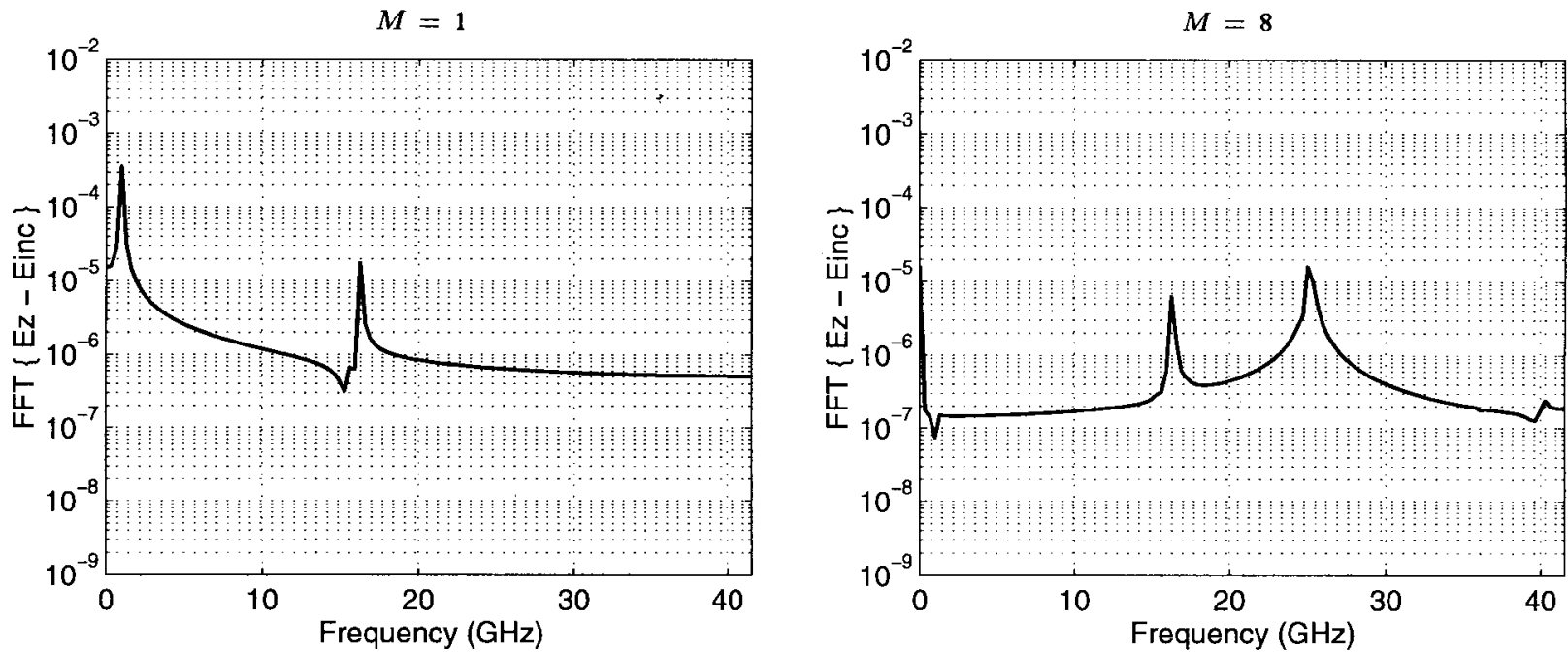

(b)

Fig. 3. Error results using a half-period-long Hanning window. The results on the left and right sides are obtained with the standard and eight-times-better sampled IFA excitations, respectively. (a) Maximum error on $E_{z}$. (b) FFT of the error on $E_{z}$ at a particular point.

\section{IMPROVING THE ACCURACY OF THE IFA EXCITATION}

If the accuracy of the IFA excitation is not enough for a certain application, the method can be modified to increase the accuracy. This modification is simply to use a finer discretization in the 1-D source grid. That is, the time and frequency sampling periods in the 1-D source grid are reduced by a fixed integer $M$. Then, $M$ of the IFA grid points fall in between two neighboring grid points in the 3-D computational domain. The geometrical view of such an increase in the sampling frequency of the IFA is depicted in Fig. 1(b), for the case of $M=4$. When the source grid is sampled $M$ times better than the 3-D grid, the 1-D finite-difference equations (1) and (2) are used $M$ times during one time step of the 3-D FDTD algorithm. Clearly, a finer sampling of the 1-D source grid results in more accurate FDTD calculations and, hence, more accurate incident-field values on the 1-D source grid. Furthermore, as the samples get denser on the source grid, the incident-field values in the 3-D grid are interpolated from closer samples and, thus, the quality of the interpolated incident-field value will be better. As a result, by using a finely sampled source grid, the accuracy of the incident-wave simulation is doubly increased. Note that the cost of the 1-D FDTD calculations on the source grid is negligible compared to the cost of the 3-D FDTD calculations in the computational domain.

In order to quantify the errors created in the plane-wave generation process and to isolate these errors from other FDTD errors, the excitation and propagation of waves in a homogeneous media are considered. A 3-D empty computational domain composed of $30 \times 30 \times 30$ Yee cells and terminated by 8 -cell-thick perfectly matched layer (PML) [12]-[14] is set up for this purpose. The PML walls are designed to have a theoretical normal reflection coefficient $R(0)$ of $10^{-4}$ and parabolic conductivity profile. The space sampling period is $\Delta=0.625 \mathrm{~cm}$. The time step is selected at the Courant stability limit as $\Delta t=12.081 \mathrm{ps}$. Separate-field formulation is employed with a total-field region of $18 \times 18 \times 18$ cells and a six-cell-thick scattered-field region. The incident plane-wave values are computed with the CFIF scheme. The plane wave 

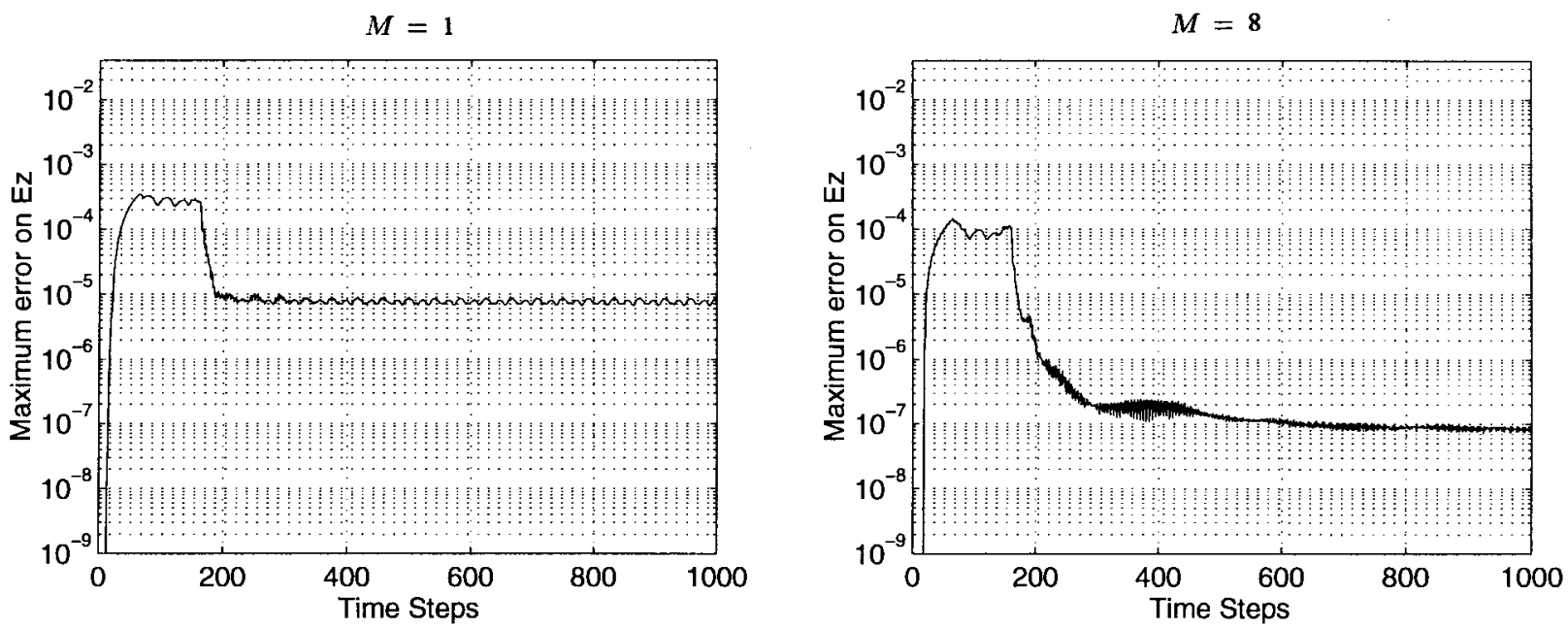

(a)
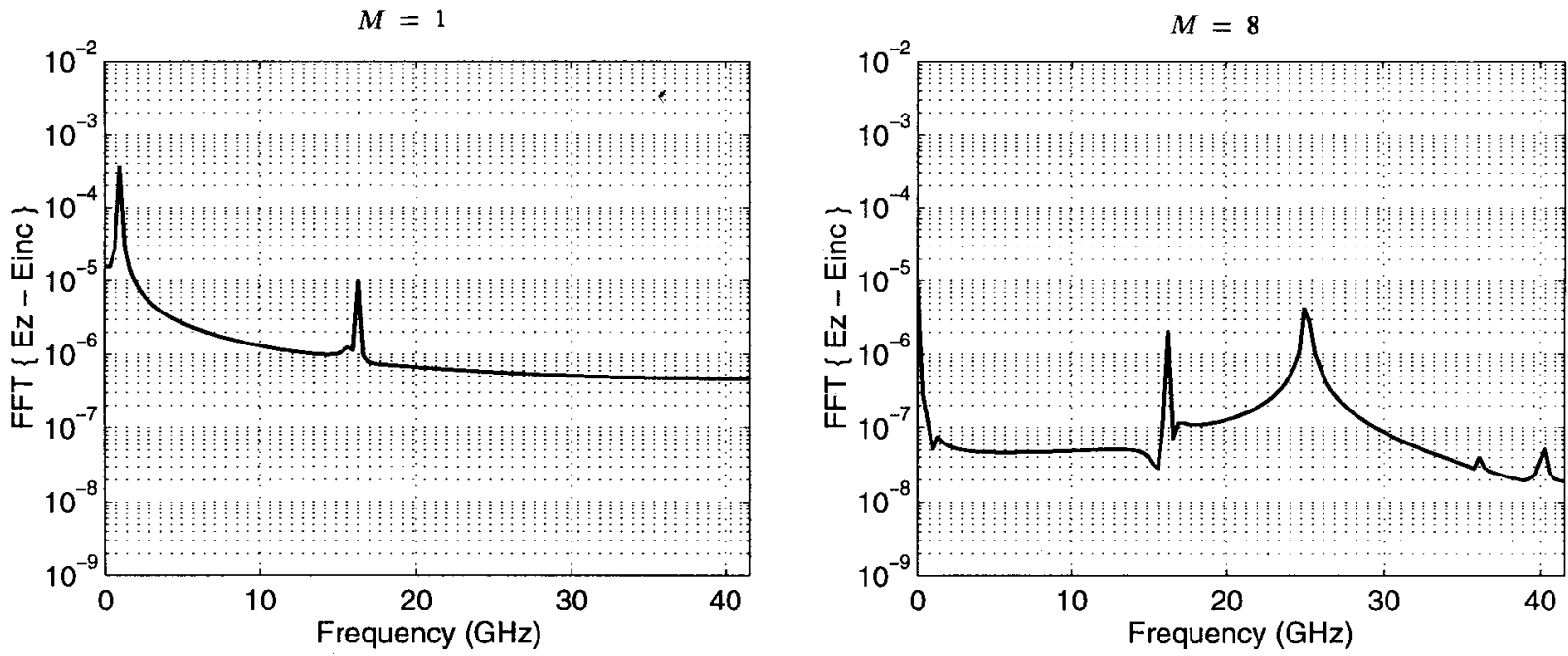

(b)

Fig. 4. Error results using a one-period-long Hanning window. The results on the left and right sides are obtained with the standard and eight-times-better sampled IFA excitations, respectively. (a) Maximum error on $E_{z}$ (b) FFT of the error on $E_{z}$ at a particular point.

is incident at $\theta=90^{\circ}$ and $\phi=45^{\circ}$. The incident electric field is polarized in the $z$-direction and its amplitude is unity. The incident magnetic field is polarized in the direction of $\hat{x}-\hat{y}$. The time dependence of the incident plane wave is given by ${ }^{1}$

$$
e(t)=w(t) \sin \left(2 \pi f_{0} t\right)
$$

where $f_{0}=1 \mathrm{GHz}$ and $w(t)$ is either the unit step function or a Hanning window defined as

$$
w(t)=\left\{\begin{array}{l}
0, \quad \text { if } t \leq 0 \\
0.5-0.5 \cos \left(\frac{\pi t}{L}\right), \quad \text { if } 0 \leq t \leq L \\
1, \quad \text { otherwise. }
\end{array}\right.
$$

Note that $w(t)$ becomes a unit step function when $L=0$. For $L>0$, the Hanning windows help reduce the FDTD

\footnotetext{
${ }^{1}$ This is not a finite-duration excitation. Therefore, physically nonzero quantities are expected to reach nonzero steady-state values for sufficiently large values of time.
}

errors due to the high-frequency components of the excitation signal by smoothing the time dependence of the incident plane wave [19].

Ideally, the fields in the total-field region of the FDTD grid should be exactly the same as the incident plane wave, and the field variables in the scattered-field region should be identically equal to zero. However, due to the approximate nature of the FDTD method, computationally obtained field variables are expected to deviate from their ideal counterparts. The deviation, i.e., the error, can be computed at each time step, in every cell, and for any field component. Fig. 2 shows the maximum value of the error in the $E_{z}$ field component over both the total-field and scattered-field domains at each time step for $M=1,4,8,16$, and 32 . These error results are obtained by using a Hanning window of length $L=$ $0.5 T_{0}$, where $T_{0}=1 / f_{0}$ is the period of the sinusoidal time dependence of the incident plane wave. The input signal is multiplied by a smoothing window at the early stages in order to decrease the errors due to the abrupt change at the onset of 

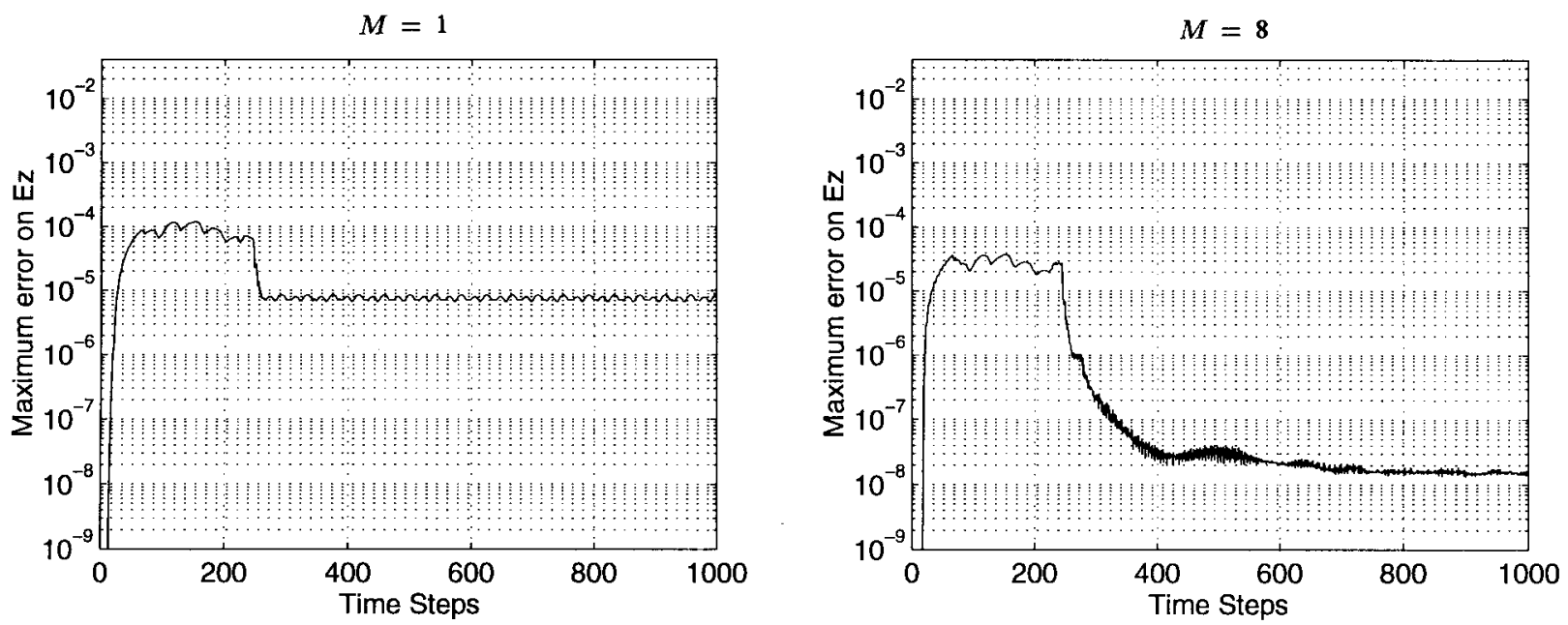

(a)
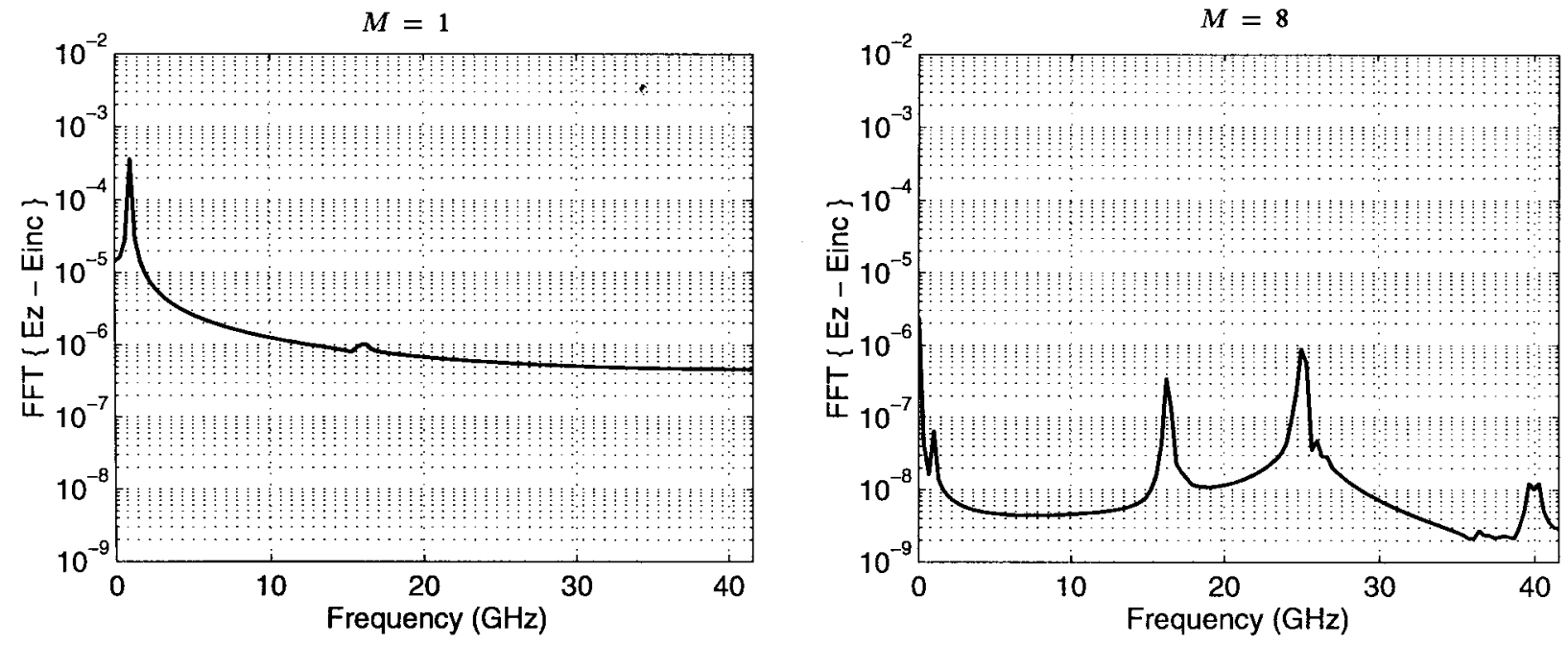

(b)

Fig. 5. Error results using a two-period-long Hanning window. The results on the left and right sides are obtained with the standard and eight-times-better sampled IFA excitations, respectively. (a) Maximum error on $E_{z}$. (b) FFT of the error on $E_{z}$ at a particular point.

the input signal, which has high-frequency components [19]. The error levels for $M \geq 4$ in Fig. 2 show great improvement with respect to the standard IFA $(M=1)$ excitation results. However, one would expect the results to get progressively better as the value of $M$ is increased. Fig. 2 shows that this is not necessarily correct, as the error levels for $M=8,16$, and 32 are actually slightly worse than that of $M=4$. The reason for this result will be explained in Sections IV and V.

Figs. 3-5 depict a careful investigation of the effect of the eight-times-better sampled IFA excitation $(M=8)$ on the FDTD errors. In Figs. 3-5, half-, one-, and two-periodlong Hanning windows are used for smoothing. In each figure, the error results for an eight-times-better sampled source grid $(M=8)$ are compared to the results of the corresponding standard IFA excitation $(M=1)$. Figs. 3(a), 4(a), and 5(a) (and other similar error plots in this paper) show the maximum value of the error in the $E_{z}$ component over both the total- and scattered-field domains at each time step. In order to have a better understanding of the nature of the error, Figs. 3(b), 4(b), and 5(b) show the frequency spectra of the error in the $E_{z}$ component at the first cell the incident wave touches in the total-field domain.

Fig. 3 shows the effects of the eight-times-better sampled IFA excitation using a half-period-long Hanning window. The maximum error level is decreased by more than one order of magnitude, as shown in Fig. 3(a). In Fig. 3(b), the error signal for the standard IFA excitation is seen to have a dominant frequency component of $1 \mathrm{GHz}$, which is significantly reduced by using eight-times-better sampling in the source grid.

In Fig. 4, the performances of the standard and the eighttimes-better sampled IFA computation schemes are compared for a full-period-long Hanning window used for smoothing. Fig. 4(a) shows that the maximum error level is decreased by almost two orders of magnitude. The 1-GHz component in the error signal is again reduced, as shown in Fig. 4(b). Fig. 5, where the results obtained with a two-period-long Hanning window are presented, is also in agreement with Figs. 3 and 4. In Fig. 5(a), increasing the sampling frequency of the source grid eight times decreases the maximum error level down to $10^{-8}$, which is almost three orders of magnitude lower than the error level obtained with the standard IFA excitation scheme. 

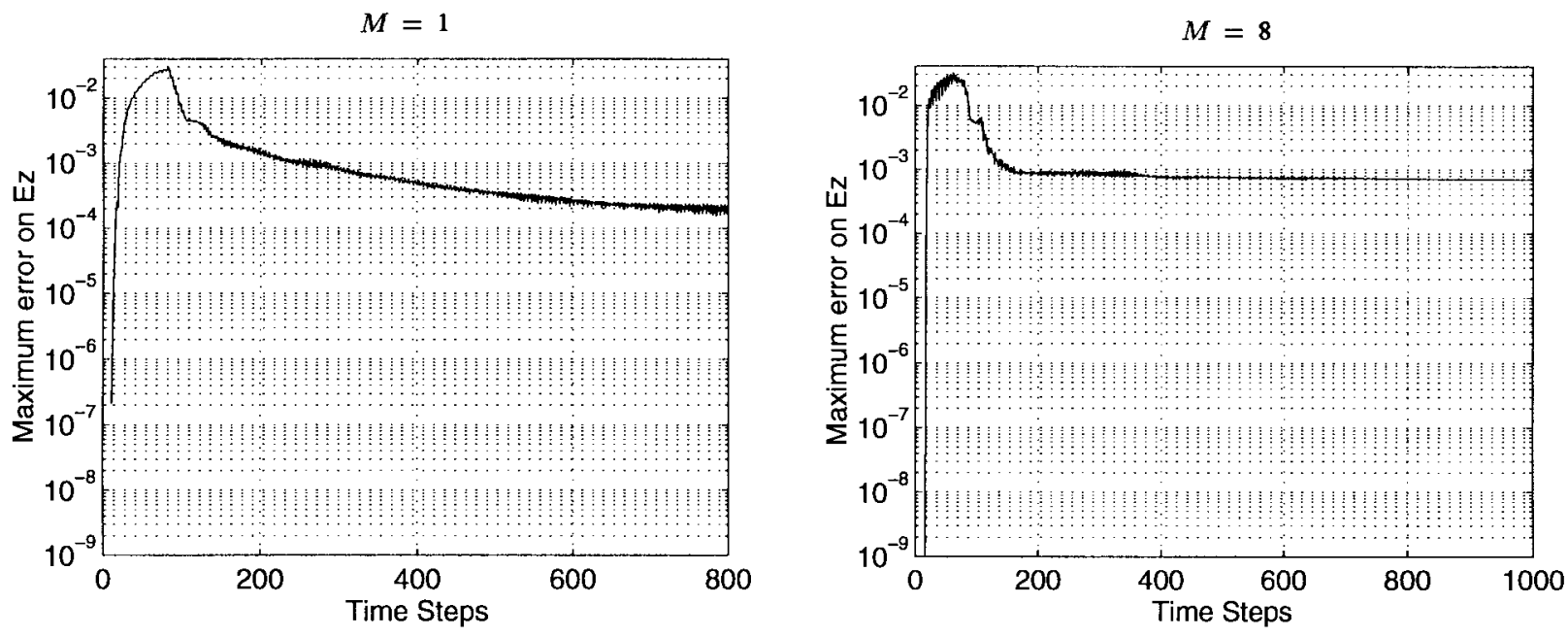

(a)
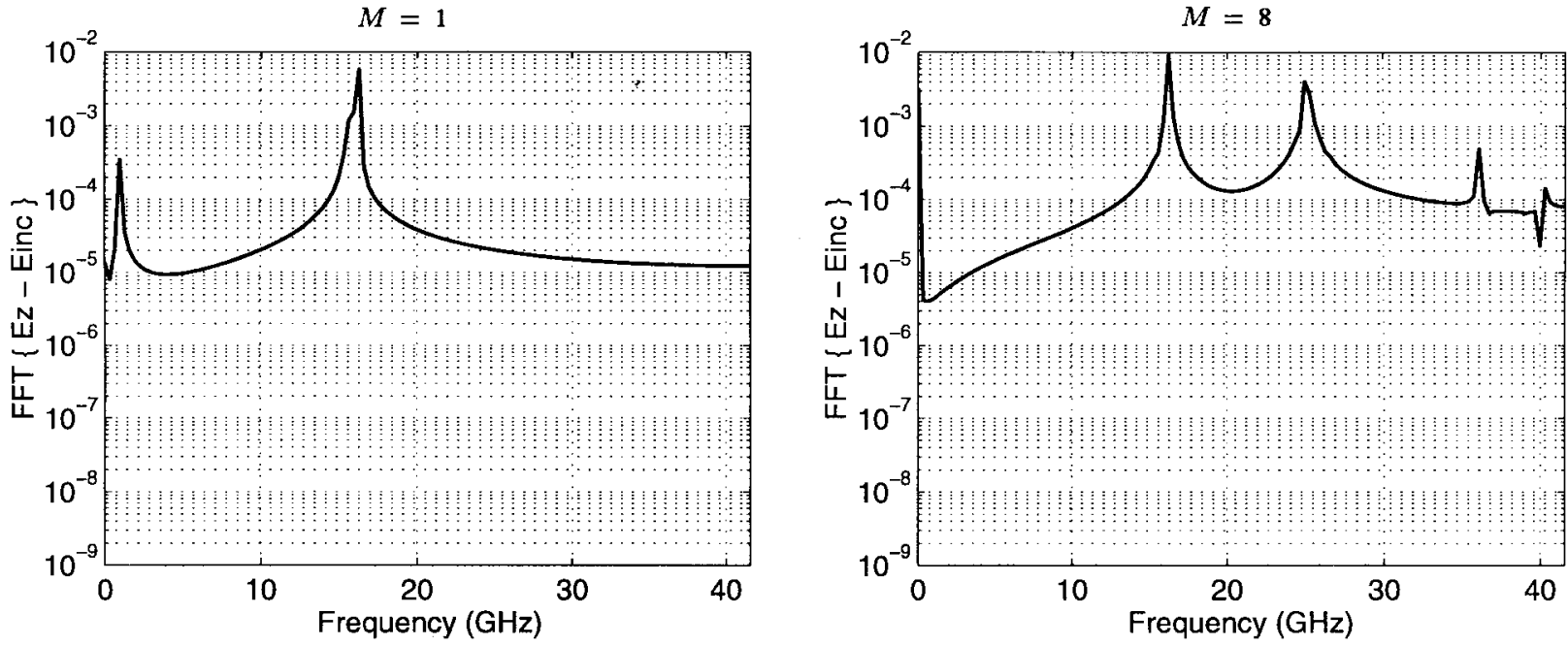

(b)

Fig. 6. Error results using no smoothing windows. The results on the left and right sides are obtained with the standard and the eight-times-better sampled IFA excitations, respectively. (a) Maximum error on $E_{z}$. (b) FFT of the error on $E_{z}$ at a particular point.

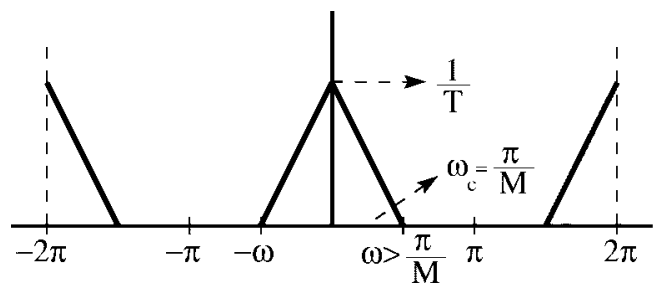

(a)

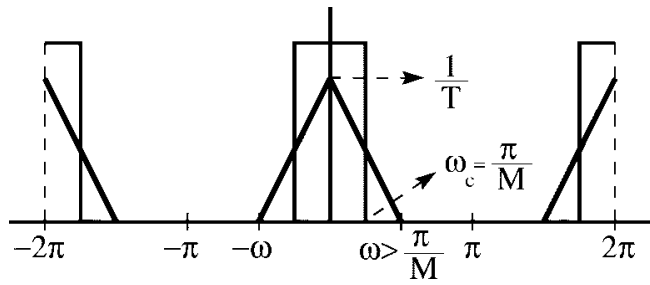

(c)

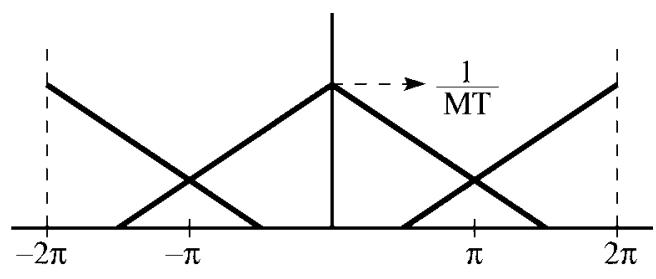

(b)

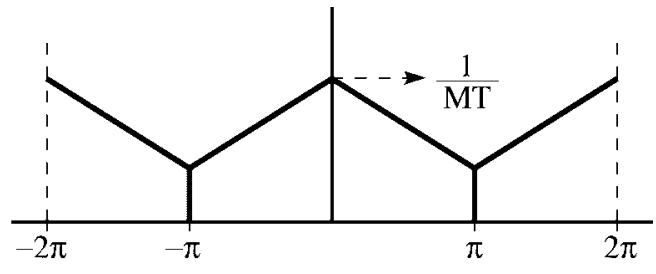

(d)

Fig. 7. (a)-(b) Downsampling with no decimation filter causes aliasing. (c)-(d) Downsampling with a decimation filter. No aliasing occurs. 


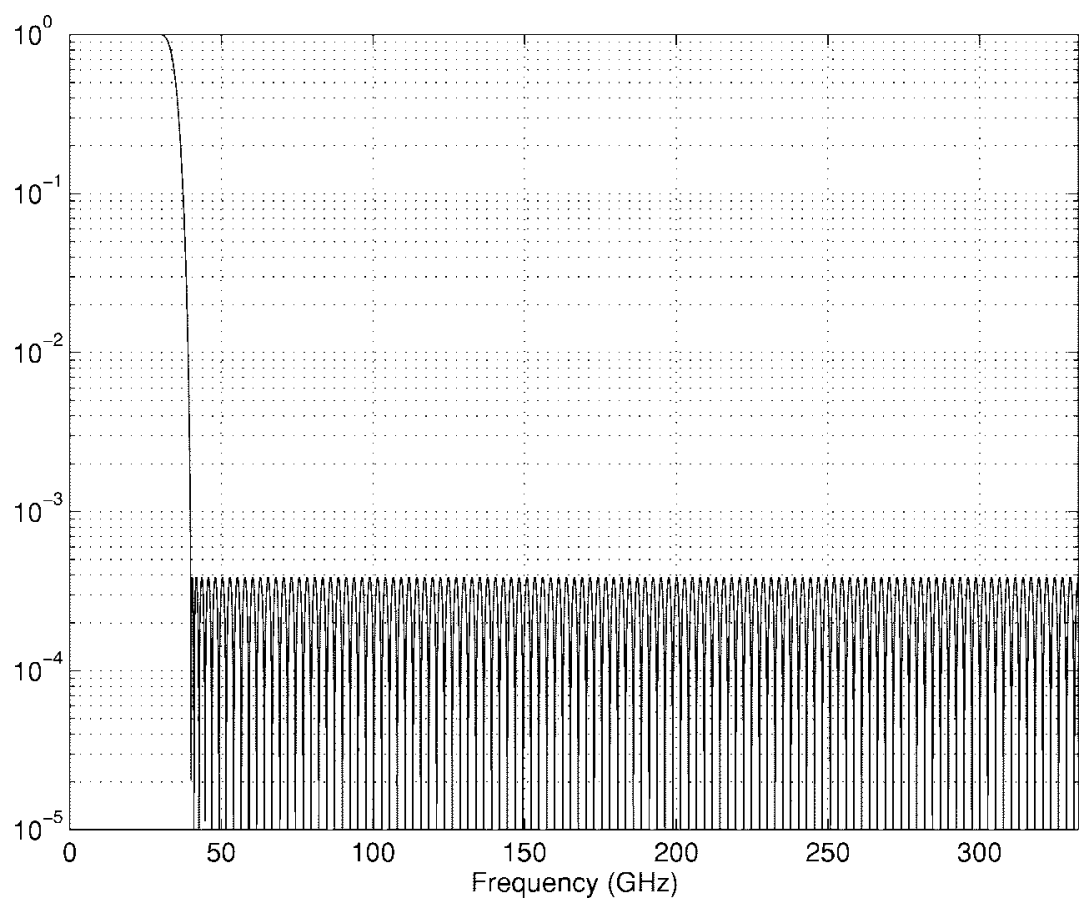

Fig. 8. The frequency response of the decimation filter with $\pi / 8$ cutoff used for eight-times-better sampled IFA excitation.

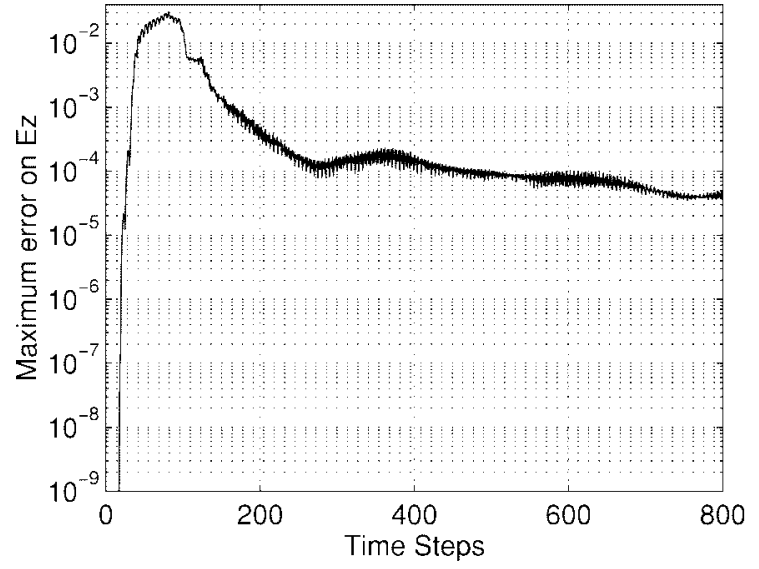

(a)

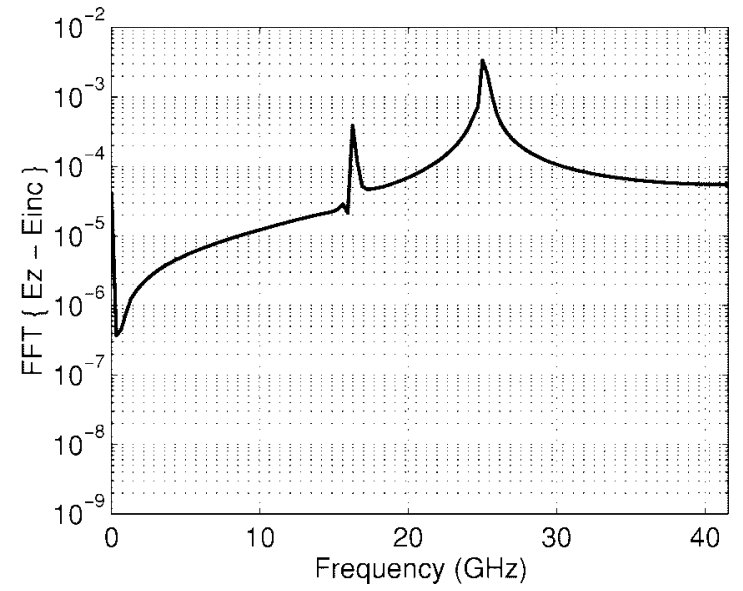

(b)

Fig. 9. Error results for the eight-times-better sampled IFA excitation with a decimation filter. No smoothing window is used. The frequency response of the 256-point digital filter is shown in Fig. 8. (a) Maximum error on $E_{z}$ in the computational domain. (b) FFT of the error signal on $E_{z}$ at a particular point.

In Figs. 3-5, the error levels are improved as the length of the smoothing window is increased for the finer source grid $(M=8)$. However, the window length does not decrease the maximum error level in the standard IFA computations $(M=1)$. In the three results with the standard IFA excitations in Figs. 3-5, the dominant frequency component of the error signal is always $1 \mathrm{GHz}$, which is the operating frequency of the incident wave. The amplitude of the 1-GHz component of the error cannot be reduced via smoothing since it is produced by the numerical dispersion due to inherent discretization of the FDTD algorithm. Thus, the 1-GHz component constitutes a threshold for the error level. This threshold can be reduced by employing a finer discretization in the FDTD method. Indeed, increasing the space and time sampling rates by eight times in Figs. 3-5 does overcome this threshold error level.
Using the same line of reasoning and noting that none of the error signals produced by the finer source grid $(M=8)$ in Figs. 3-5 has a dominant frequency component of $1 \mathrm{GHz}$, we can conclude that the error levels can be further improved. This is because the threshold error level, which is the error level of the 1-GHz component, has been reduced, and errors at other frequencies dominate the error at $1 \mathrm{GHz}$. If we can identify the sources of these other errors and reduce them, further improvements in the error levels will result, as will be discussed in the following two sections.

\section{ERrors Due to Decimation}

In the previous section, a better sampling of the source grid produced more accurate results for the simulation of the incident waves. In Figs. 3-5, the improvements obtained by 


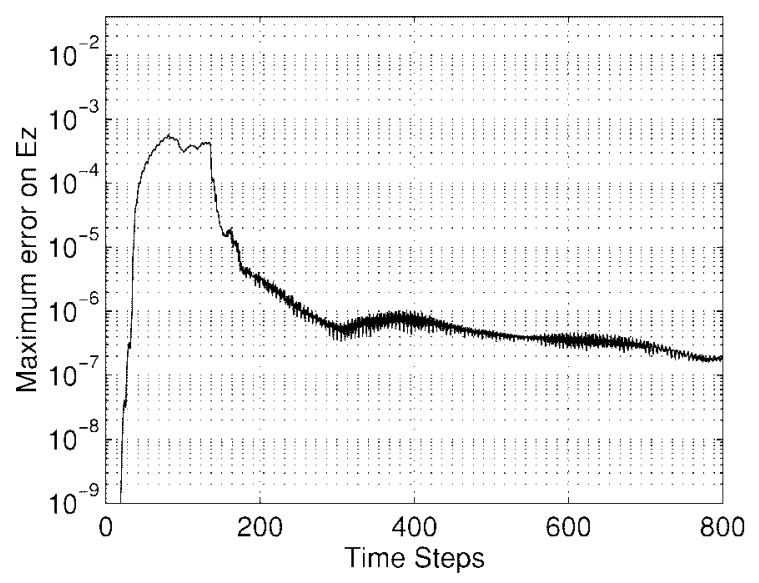

(a)

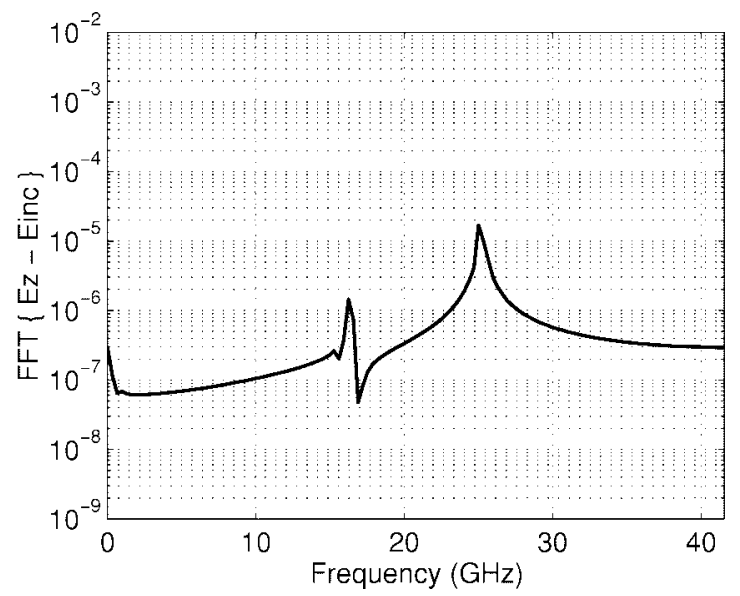

(b)

Fig. 10. Error results for the 8-times-better sampled IFA excitation with a decimation filter. A half-period-long Hanning window is used for smoothing. (a) The frequency response of the 256-point digital filter is shown in Fig. 8. (b) FFT of the error signal on $E_{z}$ at a particular point.

the better sampling of the source grid were shown. Fig. 6 depicts a similar comparison between the error results obtained with the standard and finely sampled IFA excitations when no smoothing window is used, i.e., $L=0$ in (6). Surprisingly, an improvement similar to those in Figs. $3-5$ is not observed in Fig. 6. On the contrary, the steady-state error level obtained by using an 8-times-better sampled source grid is slightly higher than that obtained by the standard IFA computation scheme, which is not expected. Furthermore, in Fig. 2, error results were presented with 4-, 8-, 16-, and 32-times-better sampled source grids, which showed that the error levels for $M=8,16$, and 32 were slightly higher than that of $M=4$. The reasons for these counterintuitive results will be explained below.

In Section III, it was explained that for an $M$ times better sampled source grid, there are $M$ points in the source grid corresponding to a single point in the 3-D FDTD grid. However, in the interpolation process, the information on these $M$ points is mapped to a single point in the computational domain. This process involves a hidden decimation or downsampling operation in it. A decimation scheme causes aliasing on the resultant signal unless a decimation filter is used before it is applied [15]. The appropriate decimation filter is a digital unity-gain low-pass filter with a normalized cutoff frequency of $\pi / M$. In Fig. 6(b), the eight-times-better sampling of the source grid produces a significant dc component in the error signal, which is absent in the result obtained with the standard IFA computation scheme with no smoothing window. This dc component hints at the presence of aliasing, which should be due to the decimation operation in this case. If this hypothesis is correct, then the reasons of the unexpected observations noted in this section can be explained. This hypothesis will be tested in the next section by using a properly designed decimation filter.

\section{USE OF A DECIMATION FILTER}

Consider the frequency-domain representation of an arbitrary digital signal, as exemplified in Fig. 7(a). The signal is sampled with a period of $T=2 \pi / \omega$ in time and a decimation of order $M$ is applied to it. However, the content of the signal is nonzero for frequencies larger than $\pi / M$. Then, the decimation produces the situation that is shown in Fig. 7(b), where the frequency components that are higher than $\pi / M$ overlap with the others and aliasing occurs. A decimation filter is needed to prevent this situation. A low-pass filter with unity gain and $\pi / M$ cutoff is applied to the signal before the decimation, as shown in Fig. 7(c). Then, the frequency components of the input signal that are higher than $\pi / M$ are suppressed. If the decimation is applied after the filter is used, then no frequency components overlap in the resultant signal, and the aliasing is prevented, as depicted in Fig. 7(d).

The signal used in the FDTD simulations is sampled with a frequency of about $83 \mathrm{GHz}$. $M$ times better sampling of the source grid means that the sampling frequency is $M \times 83 \mathrm{GHz}$ in the source grid. This frequency is normalized to $2 \pi$, as shown in Fig. 7. Then, the frequencies up to $M \times 41.5 \mathrm{GHz}$ ( $\pi$ in Fig. 7) can be resolved in the source grid. The cutoff frequency for the antialiasing filter should be at least $\pi / M$ for a decimation of order $M$. That is, the cutoff frequency of the low-pass filter should be less than or equal to $41.5 \mathrm{GHz}$.

In order to illustrate the benefits of using a decimation filter, two sinusoidal input signals, one with no smoothing and one with a half-period-long Hanning window, are sampled, filtered, and fed into the hard source of the eight-times-better sampled source grid. The frequency response of the decimation filter is shown in Fig. 8. Fig. 9 shows the error results for the filtered input with no smoothing window used. The steadystate level of the maximum error in Fig. 9(a) is more than one order of magnitude lower than the corresponding error level in Fig. 6(a). Fig. 9(b) shows that the amplitude of the dc component of the error signal is significantly reduced. Fig. 10 shows similar results for the input with a half-period-long Hanning window used together with the decimation filter. The maximum error is slightly below the corresponding error level in Fig. 3(a), where no decimation filter was used.

In Figs. 9(b) and 10(b), it is shown that the dominant frequency components are the high-frequency components around 15 and $25 \mathrm{GHz}$. It is observed that these high-frequency components adversely affect the accuracy of the results. The 


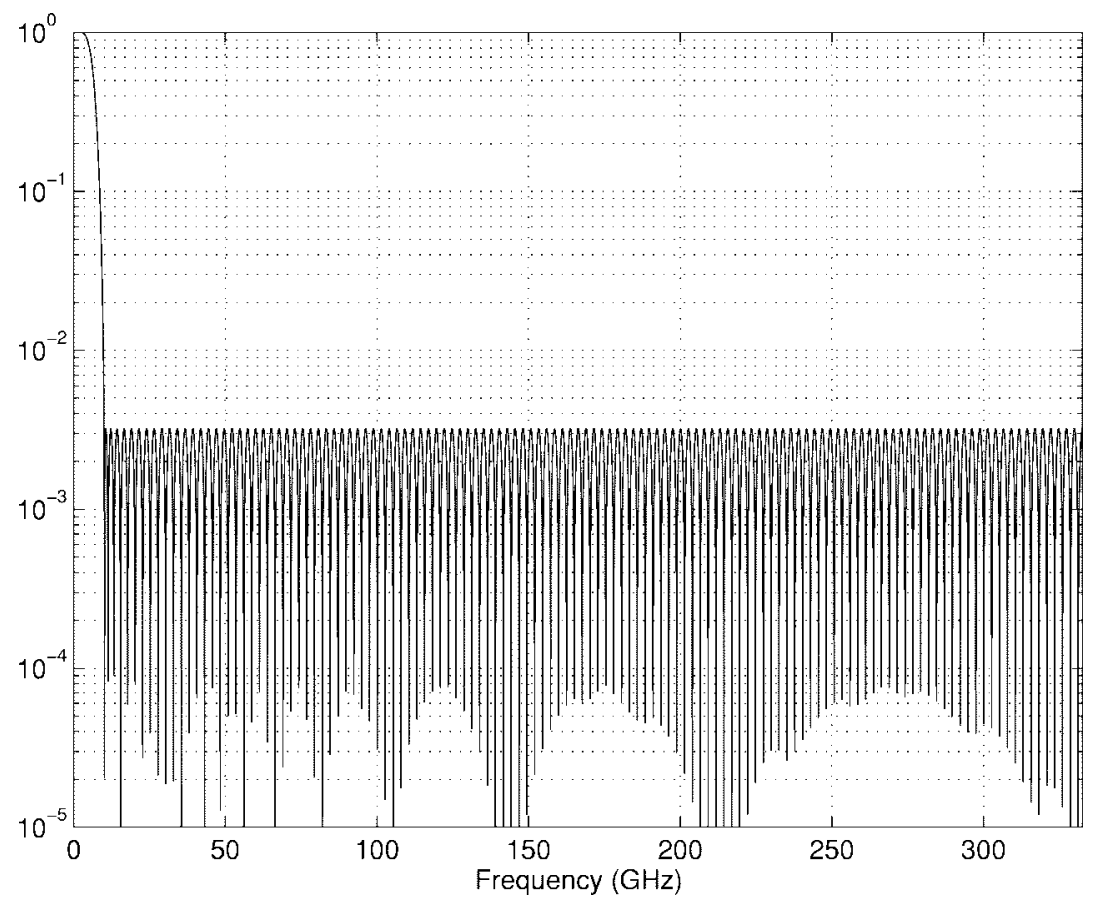

(a)

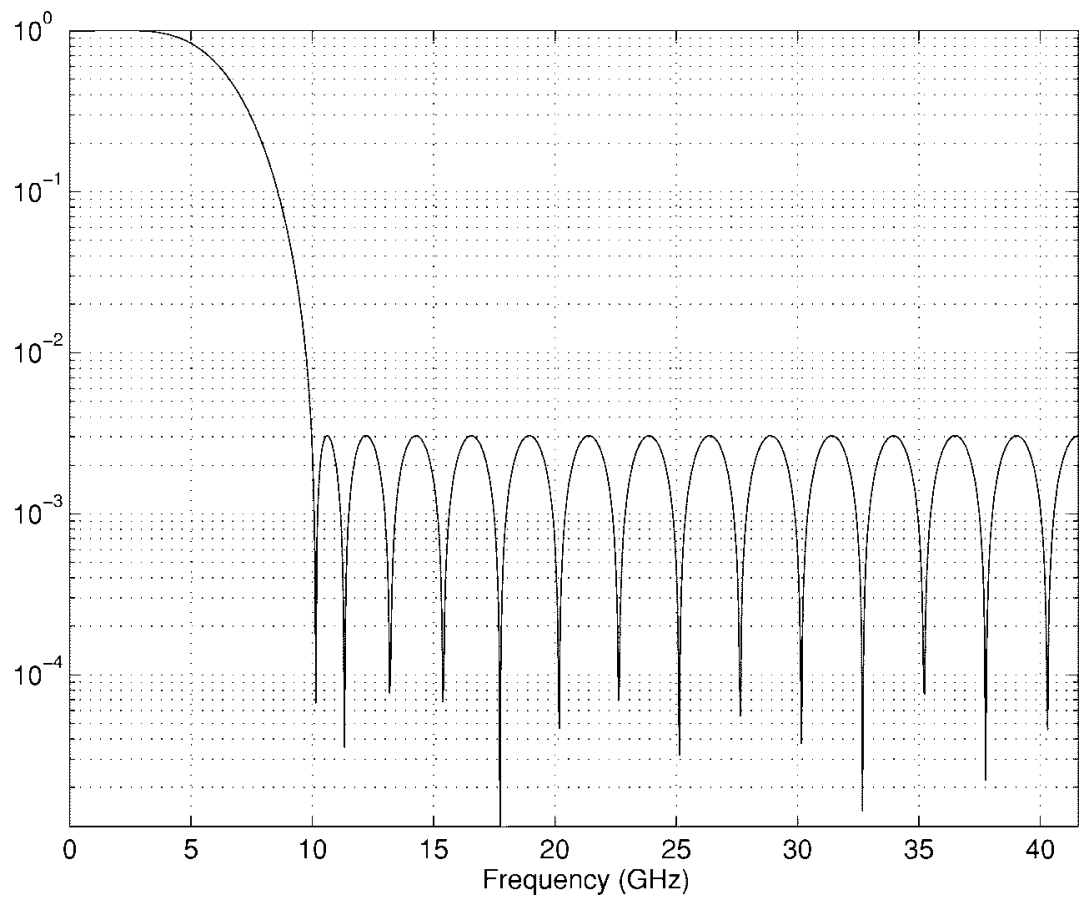

(b)

Fig. 11. (a) The frequency response of the decimation filter with $10 \mathrm{GHz}$ cutoff used for 8-times-better sampled IFA excitation. (b) An expanded view on the $0-41.5-\mathrm{GHz}$ frequency band.

cutoff frequency of the low-pass filter can be chosen in such a way as to exclude these high-frequency components from the input. Since any selection between $1-41.5 \mathrm{GHz}$ is valid, the cutoff frequency of the low-pass filter will be chosen at $10 \mathrm{GHz}$ in order to keep these undesired high-frequency components in the stopband. Fig. 11(a) shows the frequency response of this new low-pass filter. The frequency band of $0-41.5 \mathrm{GHz}$ is shown in Fig. 11(b) with an expanded view. Fig. 12 shows the error results for the input without a smoothing window, but passed through the new decimation filter. The maximum error level in Fig. 12(a) is more than two orders of magnitude lower than that of Fig. 6(a). Fig. 12(b) shows that the amplitudes of both the dc and the highfrequency components of the error signal are significantly reduced. Fig. 13 shows similar results for the input with a half-period-long Hanning window used together with the decimation filter. The maximum error is below the $10^{-8}$ level, which is about two orders of magnitude lower than the 


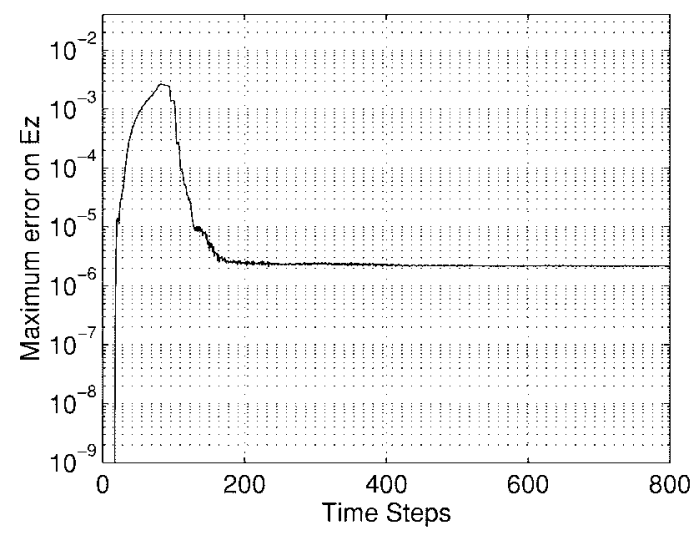

(a)

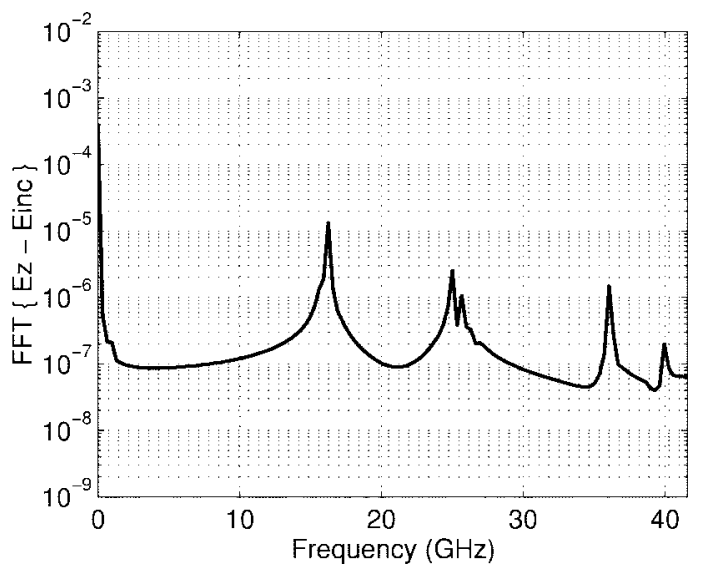

(b)

Fig. 12. Error results for the eight-times-better sampled IFA excitation with a decimation filter. No smoothing window is used. The frequency response of the 256-point digital filter is shown in Fig. 11. (a) Maximum error on $E_{z}$ in the computational domain. (b) FFT of the error signal on $E_{z}$ at a particular point.

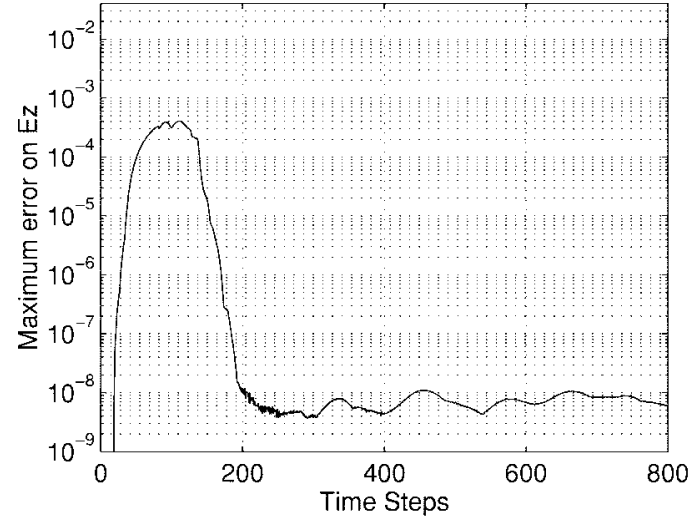

(a)

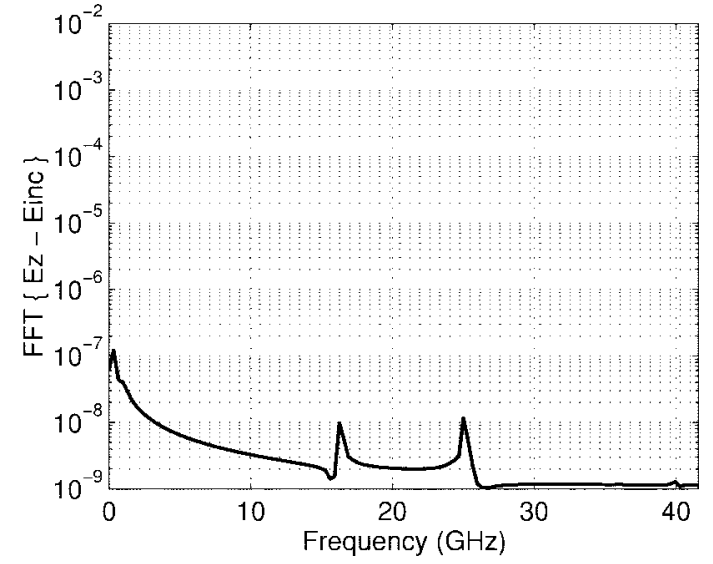

(b)

Fig. 13. Error results for the eight-times-better sampled IFA excitation with a decimation filter. A half-period-long Hanning window is used for smoothing. The frequency response of the 256-point digital filter is shown in Fig. 11. (a) Maximum error on $E_{z}$ in the computational domain. (b) FFT of the error signal on $E_{z}$ at a particular point.

corresponding error level in Figs. 3(a) and 10(a). Fig. 13(b) shows that the dominant frequency component is the $1-\mathrm{GHz}$ component, which means the maximum error level has reached the threshold for this problem. This is the ultimately desired situation in reducing the error, as explained at the end of Section III.

In Fig. 14, the maximum error levels for 4-, 8-, 16-, and 32-times-better sampled source grids are shown together. These results are obtained using the half-period-long Hanning window for smoothing and the decimation filter, shown in Fig. 11, for the prevention of aliasing due to decimation. Fig. 14 also shows the maximum error level for the standard IFA computation scheme using the Hanning window and the low-pass filter together. Since no decimation is employed for the standard IFA computation, the decimation filter does not improve the results in this case, compared to the corresponding error result in Fig. 2. The errors due to the high-frequency components are sufficiently reduced by the smoothing window and the threshold is already reached. The error results in Fig. 14 are improved to a certain extent, for the 4-timesbetter sampled source grid excitation with respect to Fig. 2, in which no decimation filter was used. Most importantly, the error levels for $M=8,16$, and 32 are below the level of $M=4$ case, contrary to Fig. 2 . The source grid resolves frequency components up to $M \times 41.5 \mathrm{GHz}$ in each excitation scheme. However, the cutoff frequency is selected as $10 \mathrm{GHz}$ in all four excitation schemes. In order to realize the same lowpass filter in wider frequency bands, longer digital filters are used. 128-, 256-, 512-, and 1024-point digital low-pass filters are designed for the 4-, 8-, 16-, and 32-times finely sampled source grid excitations, respectively [16], [17]. ${ }^{2}$

Comparing the error results presented in Figs. 3 and 13 or those in Fig. 2, one can conclude that it is possible to improve the accuracy of the incident-field excitation in the FDTD method by as much as three orders of magnitude without increasing the cost of the algorithm. This is accomplished by using a better sampled 1-D source grid in conjunction with a properly designed decimation filter.

\section{A Special Case: Normal InCidence}

In the previous sections, the performances of smoothing windows and filters have been evaluated with respect to the

${ }^{2}$ J. N. Little and L. Shure, MATLAB Signal Processing Toolbox User's Guide, MathWorks, Natick, MA, 1992. 


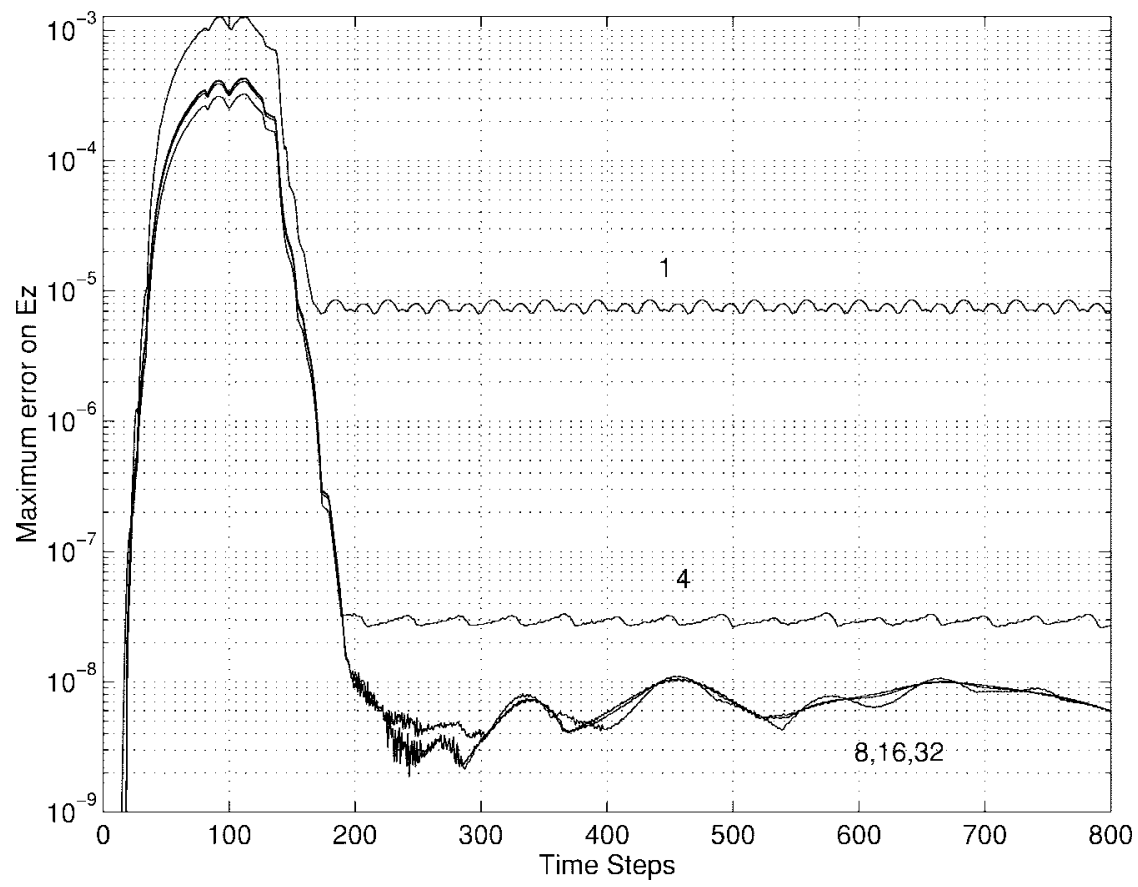

Fig. 14. The maximum errors on $E_{z}$ for 4-, 8-, 16-, and 32-times-better sampled IFA excitations with half-period-long Hanning window used together with the decimation filter. The result of the standard IFA excitation $(M=1)$ with a half-period-long Hanning window and decimation filter is also shown (labeled as " 1 ").

level of an error signal, which was defined as the difference of the total- and incident-field values. However, the FDTD algorithm may produce exactly zero error, defined in this sense, in some special cases. These special cases are the cases of the normal incidence of a plane wave in the computational domain. Normal incidence means the propagation of the wave along the $x$-, $y$-, or $z$-axis.

When the source grid is sampled with the same space and time steps as the 3-D computational domain and the direction of the incident wave is $\pm x, \pm y$, or $\pm z$, the two propagation schemes in the 1-D and 3-D grids become identical. The electric- and magnetic-field components coincide on the two grids, both in space and time. Thus, there is no need to carry out any interpolation to compute the incident-field values. These values are taken directly from the elements of the 1-D source vector. Moreover, the correction factor $\tilde{v}_{p}(\theta=0, \phi=$ $0) / \tilde{v}_{p}(\theta, \phi)$ in (1) and (2) is equal to unity for the normal incidence of the plane wave. The equations in the 3-D grid include the difference of two transversal components due to the curl operation. However, the wave has no variation in the transversal direction and the corresponding difference always vanishes. Therefore, the finite-difference update equations become identical for the field components in the 1-D source grid (IFA) and the 3-D computational grid. Since the two propagation schemes are identical, the incident wave goes through the same dispersion in the two grids, and the resultant waves are exactly the same. The scattered field is exactly zero in the scattered-field region and the difference of the total field in the total-field region and the incident field in the IFA is exactly zero. However, this does not mean that the total-field signal is exactly the same as the desired perfect sinusoid. It merely means that exactly the same discretized, thus imperfect, sinusoidal signal is propagated in the two grids.
On the other hand, when a higher sampling rate is used in the 1-D source grid, the perfect one-to-one correspondence of the 1-D and 3-D does not hold anymore. There is still no need for any interpolation, but the dispersion in the source grid is changed. Thus, the incident field on the 1-D source grid no longer exactly matches the total field in the 3-D grid. However, the quality of the total-field signal is improved in the sense that it is closer to the desired perfect sinusoid. Fig. 15 shows the difference of the amplitude ${ }^{3}$ of the total-field signal from the amplitude of a perfect sinusoid, which is unity, for three different incident-field computation schemes. In these results, the incident plane wave is the same as before, except that it propagates in the direction of $\theta=90^{\circ}, \phi=0^{\circ}$. In Fig. 15(a), the amplitude errors obtained by using the standard IFA computation scheme and a half-period-long Hanning window are shown. Increasing the sampling frequency in the source grid by eight times produces the results shown in Fig. 15(b). Since no decimation filter is used in this example, the error results are worse in Fig. 15(b) than in Fig. 15(a). However, when the decimation filter is used on the input signal before feeding into the hard source, the amplitude errors, as shown in Fig. 15(c), are decreased by about two orders of magnitude with respect to Fig. 15(a). Thus, the use of better sampled source grid together with a properly designed decimation filter helps increase the accuracy of the incident-field excitation even for the special case of normal incidence.

\section{SCATTERING ResUlts With Finer IFA ExCitATION}

The effects of improving the accuracy of the incidentfield excitation using the methods of this paper can also be demonstrated by a scattering problem. A square metal plate of

\footnotetext{
${ }^{3}$ In this paper, the amplitudes of quasi-sinusoidal signals are computed at every time step using the method outlined in [8, Appendix] and [19, Appendix].
} 


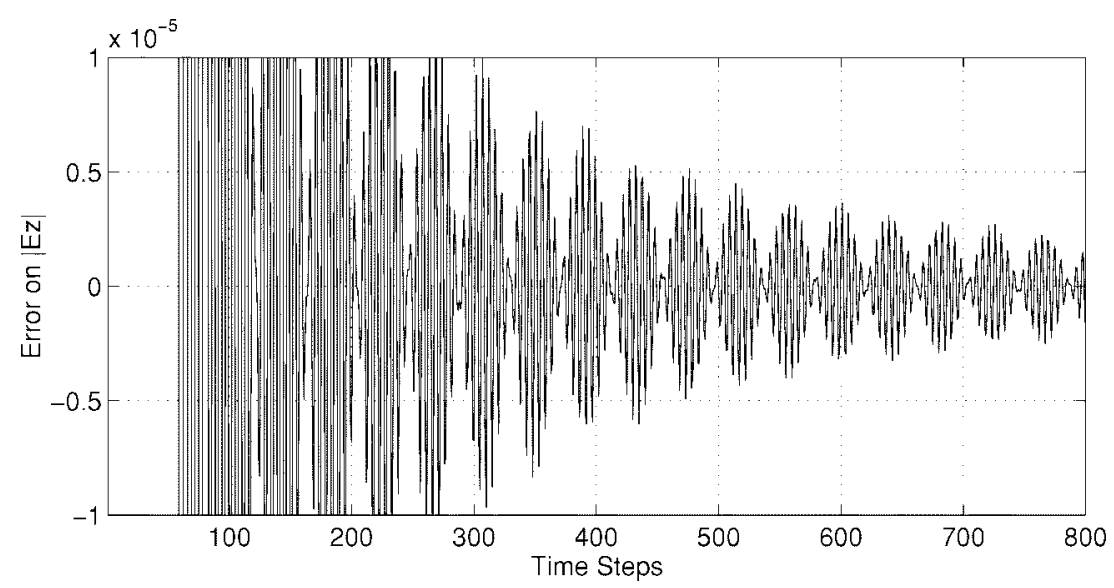

(a)

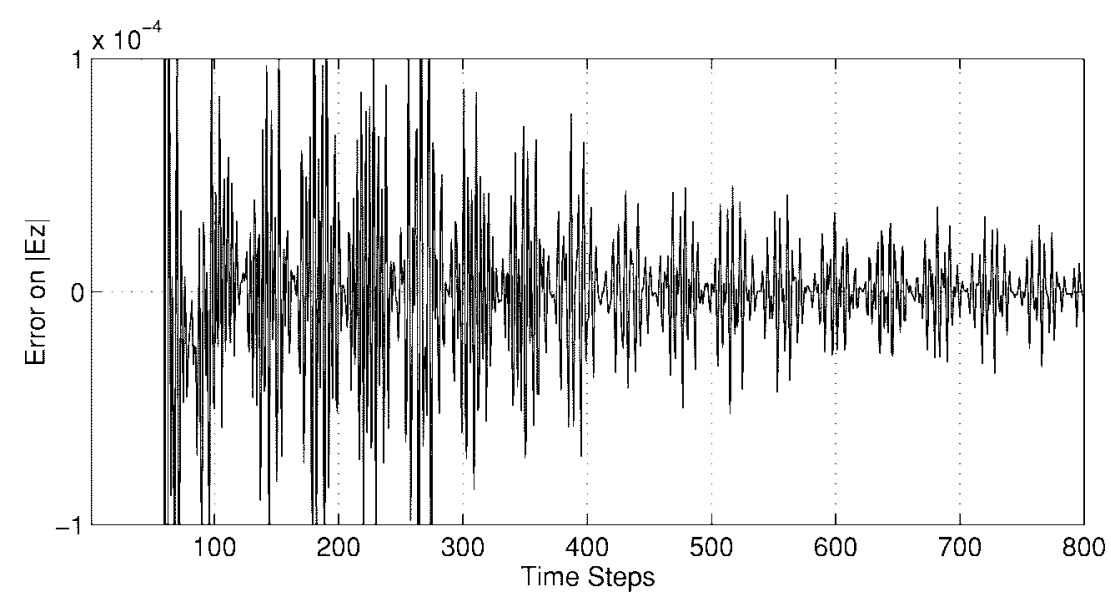

(b)

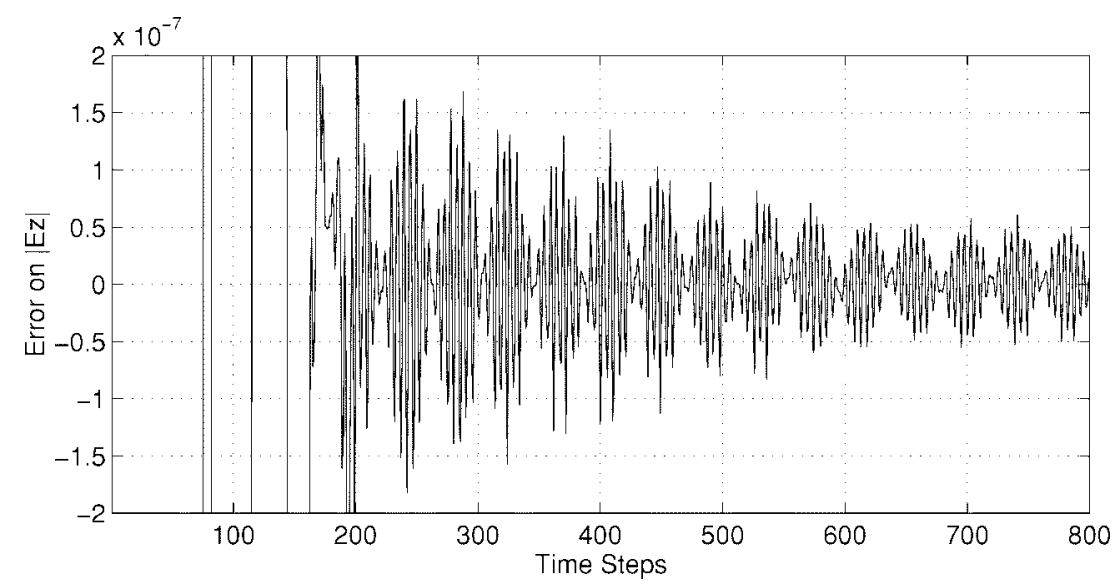

(c)

Fig. 15. Errors on the amplitude computations of $E_{z}$ at normal incidence. The three results are obtained with: (a) standard IFA excitation with half-period-long Hanning window; (b) 8-times-better sampled IFA excitation with half-period-long Hanning window; and (c) eight-times-better sampled IFA excitation with the decimation filter and a half-period-long Hanning window.

size $20 \times 1 \times 20$ Yee cells is modeled for this purpose. The plate lies on the $x-z$-plane, in the middle of a computational domain consisting of $40 \times 20 \times 40$ cells, which is divided into a total-field region of $28 \times 8 \times 28$ Yee cells and a sixcell-thick scattered-field region. The incident plane wave is identical to the one in Section III.

The $\theta$ component of the far-zone electric field $E_{\theta}$ is extrapolated at two far-zone points $(r, \theta, \phi)=\left(2000 \Delta, 90^{\circ}, 225^{\circ}\right)$ and $(r, \theta, \phi)=\left(2000 \Delta, 90^{\circ},-45^{\circ}\right)$. A time-domain farzone extrapolation scheme [18] is used for this purpose. The integration surface used for the far-zone transformation is a parallelepiped located two cells out of the total-field/scatteredfield interface. Fig. 16(a) shows the amplitude of $E_{\theta}$ at the two far-zone observation points, as computed using a standard IFA excitation scheme $(M=1)$ and a half-period-long Hanning window. Fig. 16(b) shows the corresponding results for an 

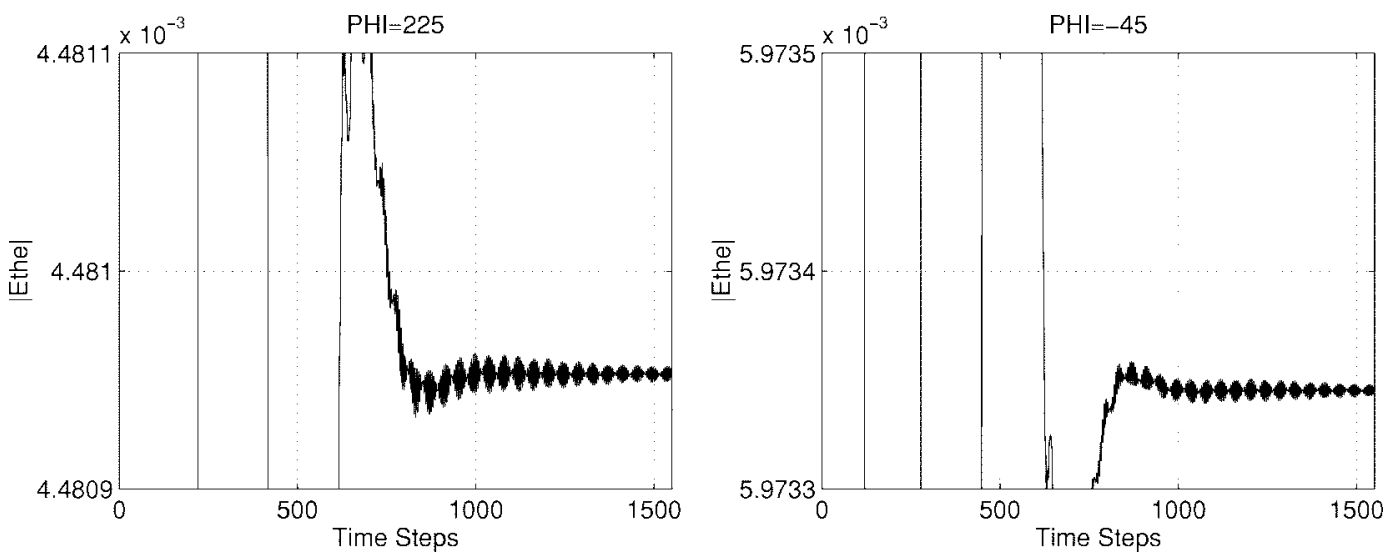

(a)
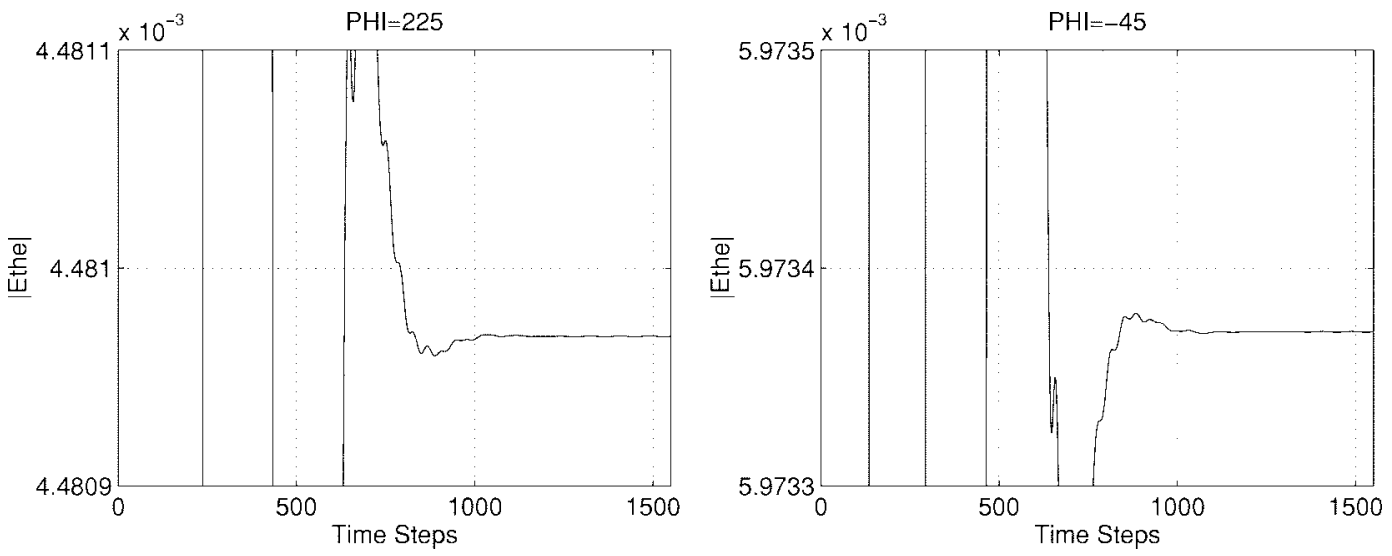

(b)

Fig. 16. Amplitude of the far-zone electric-field component $E_{\theta}$ in the directions $(\theta, \phi)=\left(90^{\circ}, 225^{\circ}\right)$ and $(\theta, \phi)=\left(90^{\circ},-45^{\circ}\right)$. The incident fields are computed with (a) standard IFA excitation $(M=1)$ and (b) 8-times-better resolved IFA excitation $(M=8)$ and a decimation filter. A half-period-long Hanning window is used for smoothing in both cases.

8-times-better sampled source grid with the same smoothing window and a decimation filter. Comparing these results, we notice that as the excitation errors are reduced, both the oscillations on the signals are diminishing and the accuracies of the steady-state levels of the signals are improving.

\section{CONCLUSIONS}

In this paper, we have presented an efficient technique to improve the accuracy of the incident-wave excitations in the FDTD calculations. The IFA excitation scheme is known to be more efficient, but less accurate, than the CFIF excitation scheme. The 1-D source grid in the IFA excitation scheme can be sampled finer to reduce the dispersion error due to discretization in the 1-D FDTD calculations, hence, to render the incident field more accurate. However, we have demonstrated that a direct application of this straightforward procedure does little or nothing to reduce the FDTD errors. Upon a careful analysis of the reasons behind this counterintuitive outcome, we have found that the interpolation operation used to transfer the incident-field values from the 1-D source grid to the 3-D computational domain behaves as an inherent downsampling or decimation process. Unless the decimated signal is sufficiently band-limited or a properly designed lowpass filter precedes the decimation operation, aliasing occurs.
Subsequently, we have been able to reduce the FDTD errors by as much as three orders of magnitude by using a decimation filter in conjunction with the finer sampling of the 1-D source grid in the IFA excitation scheme. Similar improvements have been demonstrated for the special case of normally incident waves and for a scattering problem. The technique presented in this paper can be used for the excitation of the FDTD grid by any incident wave, not just plane waves or waves with sinusoidal time dependence.

\section{ACKNOWLEDGMENT}

The authors would like to thank two anonymous reviewers for their useful suggestions and careful review of the manuscript.

\section{REFERENCES}

[1] K. S. Yee, "Numerical solution of initial boundary value problems involving Maxwell's equations in isotropic media," IEEE Trans. Antennas Propagat., vol. AP-14, pp. 302-307, Apr. 1966.

[2] A. Taflove, "Review of the formulation and applications of the finitedifference time-domain method for numerical modeling of electromagnetic wave interactions with arbitrary structures," Wave Motion, vol. 10, no. 6, pp. 547-582, 1988.

[3] A. Taflove and K. R. Umashankar, "Review of FD-TD numerical modeling of electromagnetic wave scattering and radar cross section," Proc. IEEE, vol. 77, pp. 682-699, May 1989. 
[4] K. S. Kunz and R. J. Luebbers, The Finite Difference Time Domain Method for Electromagnetics. Boca Raton, FL: CRC Press, 1993.

[5] A. Taflove, Computational Electrodynamics: The Finite-Difference Time-Domain Method. Norwood, MA: Artech House, 1995.

[6] K. L. Shlager and J. B. Schneider, "A selective survey of the finitedifference time-domain literature," IEEE Antennas Propagat. Mag., vol. 37, pp. 39-56, Apr. 1995.

[7] A. Taflove and K. Umashankar, "Radar cross section of general threedimensional scatterers," IEEE Trans. Electromag. Compat., vol. EMC25, pp. 433-440, Apr. 1983.

[8] U. Oğuz and L. Gürel, "Interpolation techniques to improve the accuracy of the plane-wave excitations in the FDTD method," Radio Sci., vol. 32, no. 6, pp. 2189-2199, Nov./Dec. 1997.

[9] G. Mur, "Absorbing boundary conditions for the finite-difference approximation of the time-domain electromagnetic-field equations," IEEE Trans. Electromag. Compat., vol. EMC-23, pp. 377-382, Apr. 1981.

[10] K. R. Umashankar and A. Taflove, "A novel method to analyze electromagnetic scattering of complex objects," IEEE Trans. Electromag. Compat., vol. EMC-24, pp. 397-405, Apr. 1982.

[11] D. E. Merewether, R. Fisher, and F. W. Smith, "On implementing a numeric Huygen's source scheme in a finite difference program to illuminate scattering bodies," IEEE Trans. Nucl. Sci., vol. 27, pp. 1829-1833, June 1980

[12] J.-P. Berenger, "A perfectly matched layer for the absorption of electromagnetics waves," J. Comput. Phys., vol. 114, no. 1, pp. 185-200, 1994.

[13] structure interaction problems," IEEE Trans. Antennas Propagat., vol. 44, pp. 110-117, Jan. 1996.

[14] _ "Three-dimensional perfectly matched layer for the absorption of electromagnetic waves," J. Comput. Phys., vol. 127, pp. 363-379, 1996.

[15] A. V. Oppenheim and R. W. Schafer, Discrete-Time Signal Processing. Englewood Cliffs, NJ: Prentice-Hall, 1989.

[16] L. R. Rabiner, J. H. McClellan, and T. W. Parks, "FIR digital filter design techniques using weighted Chebyshev approximations," Proc. IEEE, vol. 63, pp. 595-610, Apr. 1975.

[17] T. W. Parks and C. S. Burrus, Digital Filter Design. New York: Wiley, 1987.

[18] R. J. Luebbers, K. S. Kunz, M. Schneider, and F. Hunsberger, "A finitedifference time-domain near zone to far zone transformation," IEEE Trans. Antennas Propagat., vol. 39, pp. 429-433, Apr. 1991.

[19] L. Gürel and U. Oğuz, "Signal-engineering techniques to reduce the sinusoidal steady-state error in the FDTD method," Bilkent University, Ankara, Turkey, Res. Rep. BILUN/EEE/LG-9701, Mar. 1997.

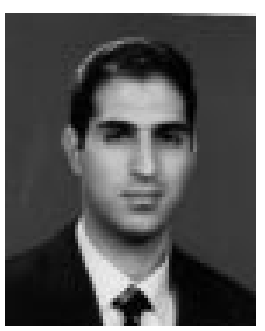

Uğur Oğuz (S'95) was born in Ankara, Turkey, on August 1, 1973. He received the B.Sc. and M.Sc. degrees in electrical and electronics engineering from Bilkent University, Ankara, Turkey, in 1994 and 1997 , respectively.

He is currently fulfilling his military obligation in Ankara, Turkey. His research interests include timedomain methods in computational electromagnetics and their applications to geophysical problems.

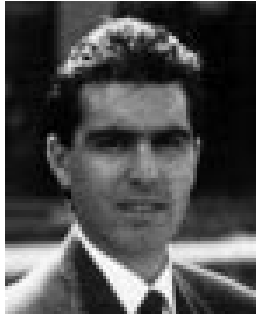

Levent Gürel (S'88-M'91-SM'97) was born in Izmir, Turkey, in 1964. He received the B.Sc. degree from the Middle East Technical University (METU), Ankara, Turkey, in 1986, and the M.S. and Ph.D. degrees from the University of Illinois at UrbanaChampaign (UIUC) in 1988 and 1991, respectively, all in electrical engineering.

In 1991, he joined the Thomas J. Watson Research Center, IBM Corporation, Yorktown Heights, NY, where he worked as a Research Staff Member on the electromagnetic compatibility (EMC) problems related to electronic packaging, use of microwave processes in the manufacturing and testing of electronic circuits, and development of fast solvers for interconnect modeling. He was awarded the title of Associate Professor by the Institute of Higher Education, Ankara, Turkey, in 1993. Since 1994, he has been a Faculty Member in the Department of Electrical and Electronics Engineering, Bilkent University, Ankara, Turkey. In 1977, he was a Visiting Associate Professor at the Center for Computational Electromagnetics (CCEM), UIUC, for one semester. His research interests include the development of fast algorithms for computational electromagnetics (CEM) and the application thereof to scattering and radiation problems involving large and complicated scatterers, antennas and radars, frequencyselective surfaces, and high-speed electronic circuits. He is also interested in the theoretical and computational aspects of electromagnetic compatibility and interference analyses. Ground penetrating radars and other subsurface scattering applications are also among his current research interests.

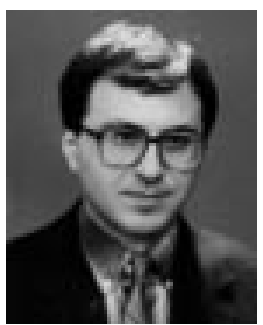

Orhan Arıkan (M'91) was born in Manisa, Turkey, in 1964. He received the B.Sc. degree in electrical and electronics engineering from the Middle East Technical University, Ankara, Turkey, in 1986, and the M.S. and Ph.D. degrees in electrical and computer engineering from the University of Illinois at Urbana-Champaign, in 1988 and 1990, respectively.

From 1990 to 1993, he was a Research Scientist at Schlumberger-Doll Research, Ridgefield, CT. Since September 1993, he has been with Bilkent University, Ankara, Turkey, where he is currently an Assistant Professor. His current research interests are in adaptive signal processing, time-frequency analyses, array signal processing, and inverse problems.

Dr. Arikan was the recipient of a Schlumberger Fellowship from 1988 to 1990. He served as the chairman of the IEEE Signal Processing Chapter, Turkey, from 1996 to 1997. 